\title{
Controlled release levodopa treatment of motor fluctuations in Parkinson's disease
}

\author{
J L JUNCOS, G FABBRINI, M M MOURADIAN, C SERRATI, A M KASK, \\ T N CHASE
}

From the Experimental Therapeutics Branch, National Institute of Neurological and Communicative Disorders and Stroke, Bethesda, Maryland, USA

SUMMARY A controlled release preparation of levodopa-carbidopa, CR III, given every 4 hours to 17 Parkinsonian patients for up to 10 months reduced plasma drug variations and improved motor response fluctuations compared with standard levodopa-carbidopa given every 2 hours. The results support the value of pharmaceutical approaches to the stabilisation of circulating levodopa levels in the treatment of Parkinson's disease.

Levodopa remains the single most effective, currently available antiParkinsonian agent. Nevertheless, disabling fluctuations in motor performance develop in many patients receiving long term treatment with this drug. ${ }^{12}$ These mainly consist of dose related wearingoff responses or the rapid, unpredictable shifts characteristic of the on-off phenomena. Previous studies have indicated that the acute maintenance of steady plasma levodopa levels, by means of intravenous drug infusions, tends to stabilise motor responses, ${ }^{3-5}$ especially in patients with wearing-off phenomena. ${ }^{67}$ More recent investigations suggest these beneficial effects may be sustained in ambulatory patients during several weeks of round-the-clock infusion. ${ }^{78}$ The foregoing observations have stimulated the search for a controlled release levodopa preparation that might provide a practical approach to the long-term maintenance of stable plasma drug levels. Those tested in the past have proven unsatisfactory, largely owing to their poor bioavailability and erratic release patterns. ${ }^{910}$ Improved formulations have recently been developed including CR III (Merck, Sharp and Dohme) in which levodopa $(200 \mathrm{mg})$ and carbidopa $(50 \mathrm{mg})$ are embedded in a non-fragmentable, polymer matrix. ${ }^{11}$ Favourable initial results with this compound $^{7}$ prompted the present study of the long term efficacy and safety of CR III in patients with wearing-off or on-off phenomena.

Address for reprint requests: TN Chase, MD. Experimental Therapeutics Branch, NINCDS/NIH, 10/5C103, 9000 Rockville Pike, Bethesda, MD 20892, USA.

Received 21 March 1986.

Accepted 25 May 1986

\section{Methods}

Seventeen patients with the diagnosis of idiopathic Parkinson's disease ( 12 men, 5 women; ages 29 to 72 years, mean 57 \pm 11 years) with symptoms of 2 to 21 years duration (mean $13 \pm 7$ years) consented to participate in this study. Parkinsonian severity (unmedicated state) ranged from mild to severe: one patient was at Hoehn and Yahr ${ }^{12}$ stage II, seven at stage III, two at stage IV, and seven at stage V. All study subjects had received standard levodopa-carbidopa (Sinemet; Merck, Sharp and Dohme) treatment (1 to 16 years, mean $10 \pm 5$ years). Seven were considered to have wearingoff responses with relatively predictable oscillations in motor performance related to their every 2 hour standard levodopa-carbidopa schedule, while 10 were diagnosed as having on-off phenomena, as defined by the presence of abrupt, erratic motor fluctuations not controllable by alterations in the timing or dosage of medication. Throughout this clinical trial two patients remained on a fixed dose of a standard anticholinergic preparation, while three continued to receive constant doses of bromocriptine or pergolide.

The study was conducted in four phases, all but the last carried out while patients were admitted to the Clinical Center at the National Institutes of Health: phase 1 -Sinemet given every 2 hours; phase 2-CR III given every 6 hours for 1 week; phase 3-CR III given every 4 hours for 1 month; phase 4 -CR III plus Sinemet (usually $50 \mathrm{mg}$ levodopa and $12.5 \mathrm{mg}$ carbidopa) given every 4 hours for 3 to 10 months. In addition, some patients also received intravenous infusions of levodopa around-the-clock in combination with orally administered carbidopa ( $50 \mathrm{mg}$ every 3 hours) for at least 3 days. All orally administered medications were given during waking hours at optimal therapeutic dose levels. Diet was controlled by restricting protein and caloric intake to the minimum daily requirement on physician rating and blood drawing days, and giving the meals at least $\mathbf{4 5}$ minutes before or after the time of drug administration. Not all patients participated in every study phase. 
Motor responses were evaluated independently by three neurologists. Overall Parkinsonian and dyskinesia severity were each scored on a scale of 0 (absent) to 5 (severe) every 20 minutes for 9 hours at the end of phases 1,2 and 3 . Simultaneous physicians' and patients' ratings were also collected. During phase 4, patients' ratings were obtained every 30-60 minutes for 9 hours for at least 4 days and then normalised to the average of the investigators' ratings.

Plasma levodopa levels were measured in blood samples collected from fully ambulatory patients through indwelling venous catheters. Samples were obtained from fasting patients for 6 hours after identical single oral doses of Sinemet or CR III; and every 20 minutes (simultaneous with physicians' ratings) for 9 hours at the end of phases 1,2 and 3 as well as during the intravenous administration of levodopa. Blood specimens were immediately centrifuged, and the plasma separated and stored at $-70^{\circ} \mathrm{C}$ until assayed by high pressure liquid chromatography with electrochemical detection. ${ }^{13}$ The relative bioavailability of levodopa was determined by measuring the area under the plasma level curves shown in fig 1 .

All patients were monitored periodically (initially weekly, subsequently bimonthly) for evidence of drug-associated changes in vital signs, or in standard haematological and blood chemistry values.

Mean $\pm S E M$ values are reported unless otherwise noted. Statistical comparison of the data employed 2-tailed, matched paired $t$ tests. Variance (computed as the square of SD values) was used as a measure of the variability of the plasma levodopa levels and motor performance scores. Interrater reliability, assessed by the intraclass correlation coefficient, was $74 \%$.

\section{Results}

Plasma levodopa levels following the oral administration of a single dose of Sinemet or of CR III differed substantially (fig 1 ). While maximum plasma

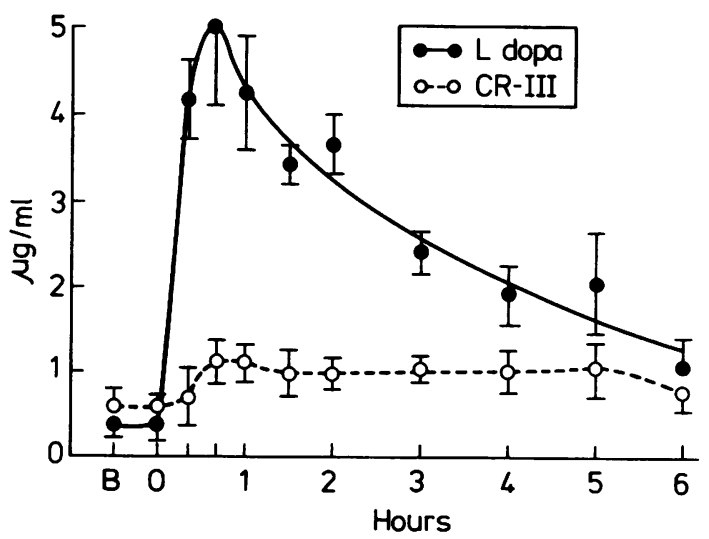

Fig 1 Comparison of plasma levodopa levels in response to equal doses of standard levodopa-carbidopa (Sinemet) or controlled release levodopa-carbidopa (CR III). Mean \pm $S E M$ values are for 5 Parkinsonian patients, at a baseline period $(B)$ and after the administration of either formulation (200 mg of levodopa plus $50 \mathrm{mg}$ of carbidopa) at time 0. levels were attained with either preparation in about 40 minutes, peak values were 9 times higher with Sinemet than with the same dose of CR III. With Sinemet, plasma levodopa levels declined with a half-life of approximately 180 minutes, while with CR III they remained essentially constant over the next 4 hours (fig 1). During the 6 hour period of this comparison, the bioavailability of levodopa, administered as $\mathbf{C R}$ III, averaged about $36 \%$ of that administered as Sinemet. Similarly, an optimal antiParkinsonian response, occurring at equivalent plasma levodopa levels, required the daily ingestion of $220 \%$ more levodopa-carbidopa when given as CR III every 6 hours than when given as Sinemet every 2 hours (table 1).

Plasma levodopa variance was significantly less with CR III given every 4 hours than with Sinemet given every 2 hours (fig 2). This decline averaged $28 \%$ and was not accompanied by any significant difference in mean levodopa levels. The intravenous administration of levodopa, compared with oral Sinemet, reduced plasma levodopa variability by $86 \%$ (fig 2). CR III was thus only about $30 \%$ as effective as intravenously administered levodopa in decreasing plasma levodopa fluctuations. Individually, nine of 10 patients converted from Sinemet to CR III manifested reductions in plasma levodopa variance ranging from 6 to $95 \%$; variance increased in the single remaining patient.

The antiParkinsonian response to CR III given every 6 hours did not differ consistently in degree or variability from that obtained with Sinemet given every 2 hours. When CR III was administered every 4 hours, however, both Parkinsonian severity and variance improved significantly (table 2 ), while dyskinesia results showed no consistent change. For the individual patient, Parkinsonian variance decreased in seven, increased in two, and remained essentially unchanged in one. In response to the relatively poor acceptability of CR III alone, especially in patients with on-off phenomena (table 3), a small supplement of Sinemet was added to the CR III regimen. With this combined therapy, Parkinsonian variance deteriorated, while Parkinsonian severity remained essentially unchanged (table 2). Patients' acceptability assessments suggested improvement in those who manifested on-off phenomena (table 3).

Patients reported the following benefits when comparing CR III with Sinemet: convenience of less frequent dosing (10 of 10 patients), diminished early morning dystonia ${ }^{14}(3$ of 3$)$ and decrease in interdose paresthesias $^{15}$ (1 of 1$)$. Improved sleep was reported in all patients studied, although the bed-time levodopa dose had to be decreased by $50 \%$ to prevent nocturnal dyskinesias $(477 \pm \mathbf{4 0} \mathrm{mg}$ compared with $240 \pm 54 \mathrm{mg} ; \mathrm{p}<0.003)$. Reported disadvantages of 
Table 1 Dose comparison of standard levodopa-carbidopa (Sinemet) with controlled release levodopa-carbidopa (CR III)

\begin{tabular}{lllll}
\hline & $\begin{array}{l}\text { Levodopa dose } \\
(\mathrm{mg} / \mathrm{kg} / \text { day })\end{array}$ & $\begin{array}{l}\text { Plasma levodopa level } \\
(\mu \mathrm{g} / \mathrm{ml})\end{array}$ & Parkinsonian score & Dyskinesia score \\
\hline Sinemet & $21 \pm 3.3$ & $6.3 \pm 1.4$ & $1.5 \pm 0.15$ & $0.75 \pm 0.13$ \\
CR III & $46 \pm 6.5^{*}$ & $4.9 \pm 1.8$ & $1.5 \pm 0.36$ & $0.97 \pm 0.35$ \\
\hline
\end{tabular}

${ }^{*} \mathrm{p}<0.001$ for difference from dose given as Sinemet.

Values are the means \pm SEM of 27 determinations obtained over a 9 hour period on each of seven Parkinsonian patients stabilised at optimal therapeutic dose levels of Sinemet (every 2 hours) and CR III (every 6 hours). Parkinsonian and dyskinesia scores are on a scale of 0 (absent) to 5 (severe).

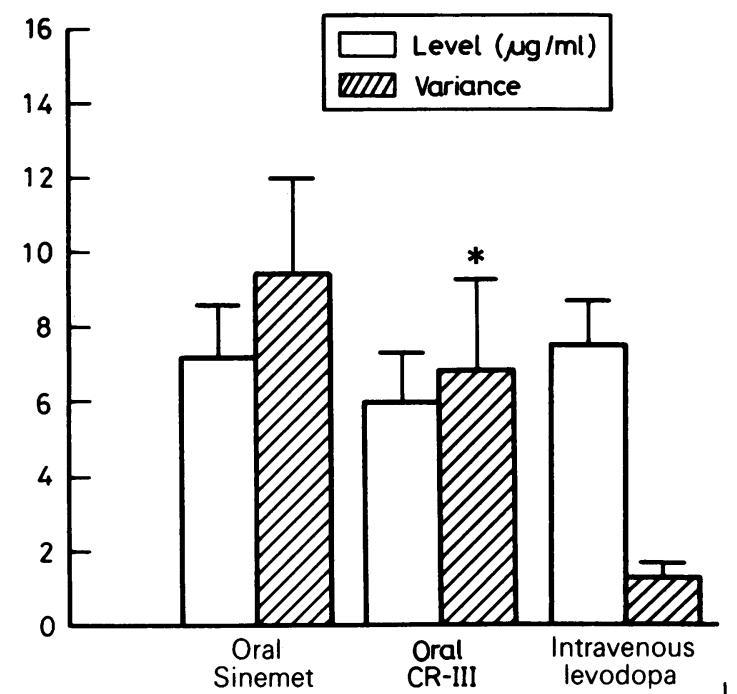

Fig 2 Comparison of controlled release levodopa-carbidopa (CR III) with standard levodopa-carbidopa (Sinemet) and intravenously administered levodopa on plasma levodopa levels and variance. Mean \pm SEM values are from 10 patients on optimal therapeutic dose levels of oral Sinemet (every 2 hours), 10 patients on CR III (every 4 hours), and 11 patients on intravenous levodopa (24 hours per day for at least 3 days in combination with oral carbidopa). ${ }^{*} p<0.05$ for percent difference in variance from oral Sinemet or intravenous levodopa.

Table 2 Comparison of antiparkinsonian response to standard levodopa-carbidopa (Sinemet) with controlled release levodopa-carbidopa (CR III) alone or with supplemented Sinemet

\begin{tabular}{lll}
\hline & Score & Variance \\
\hline Sinemet & $1 \cdot 5 \pm 0.12$ & $1 \cdot 2 \pm 0.43$ \\
CR III alone & $1 \cdot 0 \pm 0.19$ & $0.47 \pm 0.12$ \\
Percent change & $-33 \% *$ & $-60 \% *$ \\
CR III + Sinemet & $1 \cdot 0 \pm 0.13$ & $1 \cdot 1 \pm 0.29$ \\
Percent change & $-33 \% *$ & $-1 \%$ \\
\hline
\end{tabular}

* $\mathrm{p}<0.05$ based on percent change from Sinemet.

Mean \pm SEM values are based on 18 to 27 determinations for 9-10 patients over a 9 hour period during treatment with Sinemet every 2 hours, or with CR III either alone or with Sinemet every 4 hours. A negative percent change indicates improvement.
Table 3 Patient assessment of antiparkinsonian efficacy of controlled release levodopa-carbidopa (CR III) alone and in combination with Sinemet compared with Sinemet alone

\begin{tabular}{llll}
\hline & Improved & Unchanged & Worsened \\
\hline CR III alone: & & & \\
Wearing-off & 4 & 1 & 0 \\
On-off & 0 & 1 & 8 \\
CR III + Sinemet & 4 & 0 & 0 \\
Wearing-off & 4 & 1 & 1 \\
On-off & 4 & 1 & \\
\hline
\end{tabular}

Values are the number of patients reporting, arranged by type of motor fluctuations occurring with Sinemet. Ratings were made after 1 month of treatment with CR III alone (every 4 hours) and then after at least 2 months of treatment with CR III supplemented with Sinemet every 4 hours.

CR III included; lack of an obvious antiParkinsonian "surge" and difficulties with optimal dose titration. Safety data obtained during both the acute and chronic phases of this study revealed no toxic effects of CR III.

\section{Discussion}

The present results indicate that CR III functions as a controlled release levodopa preparation in Parkinsonian patients. Considering average data from the entire patient group, CR III appears to produce relatively stable plasma levodopa levels for at least 4 hours. Nevertheless, analysis of individual patient data revealed considerable residual variability in circulating drug levels. Overall the drug is only about one-third as effective as intravenously administered levodopa in reducing the fluctuations in plasma levodopa which attend the oral administration of Sinemet. Moreover, the bioavailability of CR III is relatively limited, averaging about one-third that of Sinemet. An oral intake more than twice that of the standard drug is thus necessary to achieve comparable blood levels and antiParkinsonian efficacy. Variations in plasma levodopa with CR III probably can not be attributed to dietary influences, since these were minimised during days when circulating drug levels were measured. Gastrointestinal factors are more likely to have been contributory, since abnormalities in this system are common in Parkinsonian 
patients. ${ }^{516}$ The nonfragmentable matrix used in the formulation of CR III may also have been partly responsible for the plasma levodopa variations noted in the present study.

CR III given every 4 hours significantly reduced the motor fluctuations observed during treatment with Sinemet given every 2 hours. Although these variations improved in a majority of patients, the degree of benefit was often small and inconsistent from dose to dose. The objective findings revealed a statistically significant improvement only in Parkinsonian scores and variance values; our failure to document a statistically significant reduction in dyskinesia variance presumably reflects the large intra- and interpatient variability, small number of study subjects, and the modest overall drug effect. Since stabilisation of plasma levodopa levels through its intravenous administration diminishes the motor fluctuations in most Parkinsonian patients, ${ }^{3-8}$ the relatively modest reductions in plasma levodopa variance achieved with CR III may have contributed to the minor clinical improvement observed in most patients admitted to this study. Furthermore, the limited efficacy of CR III probably also reflected the advanced stages of illness of many study subjects.

Some patients, especially those with on-off phenomena, did not perceive sufficient improvement in response stability with CR III to offset the loss of the antiParkinsonian surge typically associated with Sinemet. In an attempt to correct this deficiency, a small supplement of Sinemet was added to each patient's CR III regimen. As a result, Parkinsonian fluctuations increased, while CR III acceptance by patients with on-off phenomena improved. This apparent paradox may be attributable to the brief elevation in circulating drug levels produced by levodopa supplementation, thus increasing plasma levodopa variance and providing a brisk antiParkinsonian effect to those patients whose motor responses did not completely stabilise on CR III.

In terms of patient acceptability, CR III had several advantages over Sinemet. These benefits included the convenience of less frequent dosing, diminished early morning dystonia, and improved sleep. A major disadvantage of CR III, especially in patients with on-off phenomena, was the perceived lack of a reliable antiParkinsonian surge following drug ingestion. Attempts to increase the dose or shorten the interdose interval failed to improve antiParkinsonian efficacy and often resulted in prolonged periods of severe dyskinesia or akinesia.

CR III appears to be a modest, yet definite, step forward in the formulation of drugs for the relief of Parkinsonian symptoms. More importantly, observations with this drug support the concept that pharmaceutical attempts to stabilise plasma levodopa fluctuations will tend to smooth the motor response fluctuations which complicate standard levodopa therapy. Levodopa toxicity, as well as disease progression, may contribute significantly to the presence of these response fluctuations by several mechanisms. $^{817}$ It is tempting to speculate that the continuing oscillations in circulating plasma and presumably brain levels of levodopa attending the administration of standard oral preparations may have pathogenetic significance. This possibility is supported by reports that bromocriptine, with a plasma half life more than twice that of levodopa, is less prone to produce motor response fluctuations. ${ }^{18}$ Studies of the prophylactic value of sustained release preparations in less incapacitated patients may thus be of interest. In the meantime, the present results should stimulate further attempts to develop controlled release formulations with improved bioavailability and more stable release patterns.

\section{References}

1 Fahn S. "On-off” phenomenon with levodopa therapy in Parkinsonism. Clinical and pharmacologic correlations and the effect of intramuscular pyridoxine. Neurology 1974;24:431-41.

2 Marsden CD, Parkes JD. "On-off' effects in patients with Parkinson's disease on chronic levodopa therapy. Lancet 1976;1:292-6.

3 Shoulson I, Glaubiger GA, Chase TN. On-off response. Clinical and biochemical correlations during oral and intravenous levodopa administration in Parkinsonian patients. Neurology 1975;25:1144-8.

4 Quinn N, Parkes JD, Marsden CD. Control of on/off phenomenon by continuous intravenous infusion of levodopa. Neurology 1984;34:1131-6.

5 Nutt JG, Woodward WR, Hammerstad JP, Carter JH, Anderson JL. The "On-off' phenomenon in Parkinson's disease. Relation to levodopa absorption and transport. N Engl J Med 1984;310:483-8.

6 Hardie RJ, Lees AJ, Stern GM. On-off fluctuations in Parkinson's disease. A clinical and neuropharmacological study. Brain 1984;107:487-506.

7 Juncos JL, Serrati C, Fabbrini G, Chase TN. Fluctuating levodopa concentrations and Parkinson's disease. Lancet 1985;2:440.

8 Chase TN, Juncos JL, Serrati C, Fabbrini G, Bruno G. Fluctuations in response to chronic levodopa therapy. Pathogenetic and therapeutic considerations. In: Yahr M, Bergmann K, eds. Parkinson's Disease. New York: Raven Press, 1986;45;477-80.

9 Woods AC, Glaubiger GA, Chase TN. Sustained release levodopa. Lancet 1973;1:1391.

10 Curzon G, Friedel J, Grier L, Marsden CD, Parkes JD, Shipley M, Zilkha KJ. Sustained-release levodopa in Parkinsonism. Lancet 1973;1:431.

11 Hutton JT, Dippel RL, Bianchine JR, Strahlendorf HK, Meyer PG. Controlled-release carbidopa/levodopa in the treatment of Parkinsonism. Clin Neuropharmacol 1984;7(2):135-9. 
12 Hoehn MM, Yahr MD. Parkinsonism: onset, progression and mortality. Neurology 1967;17: 427-442.

13 Wagner J, Vitali P, Palfreyman MG, Zraika M, Huot S. Simultaneous determination of 3,4-dihydroxyphenylalanine, 5-hydroxytryptophan, dopamine, 4-hydroxy-3-methoxyphenylalanine, norepinephrine, 3,4-dihydroxyphenylacetic acid, homovanillic acid, serotonin, and 5-hydroxyindoleacetic acid in rat cerebrospinal fluid and brain by high-performance liquid chromatography with electrochemical detection. J Neurochem 1982;38:1241-54.

14 Melamed E. Early-morning dystonia. A late side effect of long-term levodopa therapy in Parkinson's disease.
Arch Neurol 1979;36:308-10.

15 Nutt JG, Carter JH. Sensory symptoms in Parkinsonism related to central dopaminergic function. Lancet 1984;ii:465-7.

16 Eadie MJ, Tyrer JH. Alimentary disorders in parkinsonism. Aus Ann Med 1965;14:13-27.

17 Lesser RP, Fahn S, Snider SR, Cote LJ, Isgreen WP, Barrett RE. Analysis of the clinical problems in Parkinsonism and the complications of long-term levodopa therapy. Neurology 1979;29:1253-60.

18 Lees AJ, Stern GM. Sustained bromocriptine therapy in previously untreated patients with Parkinson's disease. J Neurol Neurosurg Psychiatry 1981;44:1020-3. 